\title{
HYPONORMAL OPERATORS QUASISIMILAR TO AN ISOMETRY ${ }^{1}$
}

\author{
BY
}

PEI YUAN WU

\begin{abstract}
An expression for the multiplicity of an arbitrary contraction is presented. It is in terms of the isometries which can be densely intertwined to the given contraction. This is then used to obtain a generalization of a result of Sz.-Nagy and Foiaş concerning the existence of a $C_{.}{ }_{0}$ contraction which is a quasiaffine transform of a contraction. We then consider the problem when a hyponormal operator is quasisimilar to an isometry or, more generally, when two hyponormal contractions are quasisimilar to each other. Our main results in this respect generalize previous ones obtained by Hastings and the author. For quasinormal and certain subnormal operators, quasisimilarity or similarity to an isometry may even imply unitary equivalence.
\end{abstract}

1. Introduction. Let $T_{1}$ and $T_{2}$ be bounded linear operators on the complex, separable Hilbert spaces $H_{1}$ and $H_{2}$, respectively. We say that $T_{1}$ is densely intertwined to $T_{2}\left(T_{1} \prec T_{2}\right)$ if there is a bounded linear transformation $X: H_{1} \rightarrow H_{2}$ with dense range such that $X T_{1}=T_{2} X ; T_{1}$ is a quasiaffine transform of $T_{2}\left(T_{1} \prec T_{2}\right)$ if the intertwining operator $X$ is a quasiaffinity, that is, it has trivial kernel and dense range. We say that $T_{1}$ is densely similar to $T_{2}\left(T_{1} \stackrel{d}{\sim} T_{2}\right)$ if $T_{1} \stackrel{d}{\prec} T_{2}$ and $T_{2} \stackrel{d}{\prec} T_{1} ; T_{1}$ is quasisimilar to $T_{2}\left(T_{1} \sim T_{2}\right)$ if $T_{1} \prec T_{2}$ and $T_{2} \prec T_{1}$. The multiplicity $\mu_{T}$ of an operator $T$ on $H$ is the minimum cardinality of a set $K \subseteq H$ for which $H=\bigvee_{n=0}^{\infty} T^{n} K$. In $\S 2$, we first show that the multiplicity of an arbitrary contraction $T$ is equal to the minimum multiplicity of an isometry which can be densely intertwined to $T$ (Theorem 2.6). This result is inspired by the recent work of Alexander [1] on the quasisimilarity of a contraction to a unilateral shift.

Recall that the defect indices of a contraction $T$ are $d_{T}=\operatorname{rank}\left(1-T^{*} T\right)^{1 / 2}$ and $d_{T^{*}}=\operatorname{rank}\left(1-T T^{*}\right)^{1 / 2} . T$ is of class $C_{.0}$ (resp. $C_{0}$.) if $T^{* n} x \rightarrow 0$ (resp. $T^{n} x \rightarrow 0$ ) for all $x ; T$ is of class $C_{\cdot 1}$ (resp. $C_{1}$.) if $T^{* n} x \rightarrow 0$ (resp. $T^{n} x \rightarrow 0$ ) for all $x \neq 0$. $C_{\alpha \beta}=C_{\alpha} . \cap C_{. \beta}$ for $\alpha, \beta=0,1$. A contraction $T$ can be decomposed as $U_{s} \oplus U_{a} \oplus$ $T^{\prime}$, where $U_{s}$ and $U_{a}$ are singular and absolutely continuous unitary operators and $T^{\prime}$ is completely nonunitary (c.n.u.); $U_{s} \oplus U_{a}$ and $T^{\prime}$ are called the unitary part and c.n.u. part of $T$, respectively. Following Alexander [1], we say that the contraction $T$ is of analytic type if it has no singular unitary direct summand. For such a

Received by the editors October 10, 1984.

1980 Mathematics Subject Classification. Primary 47B20; Secondary 47A45, 47B35.

Key words and phrases. Contraction, unilateral shift, contraction of analytic type, isometry, quasisimilarity, multiplicity, hyponormal operator, quasinormal operator, Toeplitz operator.

${ }^{1}$ This research was partially supported by National Science Council (Republic of China). 
contraction $T$, the functional calculus $\varphi(T)$ for $\varphi \in H^{\infty}$ is well defined. For the details and other properties of contractions, readers are referred to Sz.-Nagy and Foiaş' book [17]. As a corollary to our preceding result, we show that if $T$ is an analytic type contraction, then there exists a $C_{._{0}}$ contraction $V$ with $d_{V^{*}}=\mu_{V}=\mu_{T}$ such that $V \prec T$ (Theorem 2.5). This generalizes the main result in [16], where $T$ is only assumed to be of class $C_{._{0}}$.

In $\S 3$, we turn to the question when a hyponormal operator is quasisimilar to an isometry or, more generally, when two hyponormal contractions are quasisimilar to each other. These questions have been considered before by Hastings [10, 11] and Conway [3] for certain subnormal operators and the author [20] for hyponormal contractions with finite defect indices. We generalize some of these previous results by showing that if $T$ and $S$ are hyponormal contractions one of whose c.n.u. parts is of finite multiplicity, then $T$ is quasisimilar to $S$ if and only if their unitary parts are unitarily equivalent and their c.n.u. parts are quasisimilar to each other (Corollary 3.10). Our strategy for the proof is similar to the one employed in [20]. The assumption on hyponormality is needed only through the fact that a hyponormal contraction can be decomposed as the direct sum of a $C_{11}$ contraction (its unitary part) and a $C_{.0}$ contraction (its c.n.u. part) (cf. [15]). Hence we can consider the quasisimilarity of such more general contractions. By exploiting the maximality of the "canonical isometry" of a $C_{1}$. contraction, we prove that if $T$ is a $C_{1}$. contraction with finite multiplicity and $X \in\{T\}$ ', the commutant of $T$, has dense range, then $X$ must be one-to-one (Theorem 3.7). This is then used to prove our main result as in [20].

Finally, in $\$ 4$ we restrict ourselves to quasinormal and subnormal operators. We show that, in certain circumstances, quasisimilarity or similarity to an isometry may imply unitary equivalence. For example, we show that a quasinormal operator quasisimilar to an isometry is unitarily equivalent to it (Proposition 4.2) and that if $V$ is an isometry and $T \in \operatorname{Alg} V$, the weakly closed algebra generated by $V$ and $I$, then $T \approx V$ implies $T \cong V$ (Proposition 4.6). The case when $V$ is a simple unilateral shift and $T \sim V$ has been considered by Conway [5].

In this paper, we use $T_{1} \approx T_{2}$ to denote that $T_{1}$ is similar to $T_{2}$ and $T_{1} \cong T_{2}$ to denote $T_{1}$ is unitarily equivalent to $T_{2}$.

2. Multiplicity of contractions. We start with the following lemma whose proof is left to the readers.

LEMMA 2.1. Let $T_{1}$ and $T_{2}$ be operators on $H_{1}$ and $H_{2}$, respectively.

(a) If $T_{1} \stackrel{d}{\prec} T_{2}$, then $\mu_{T_{1}} \geqslant \mu_{T_{2}}$.

(b) If $T_{1} \stackrel{d}{\sim} T_{2}$, then $\mu_{T_{1}}=\mu_{T_{2}}$.

For $\alpha=1,2, \ldots, \infty$, let $S_{\alpha}$ denote the unilateral shift of multiplicity $\alpha$ on $H_{\alpha}^{2}$. A simple unilateral shift is one with multiplicity one. 
LEMMA 2.2. Let $T$ be a contraction of analytic type. Then $S_{\infty} \stackrel{d}{\prec} T$.

Proof. If $V$ is the minimal isometric dilation of $T$, then $V \stackrel{d}{\prec} T$ (cf. [17, Theorem I.4.1]). Since $T$ is of analytic type, $V$ is the direct sum of an absolutely continuous unitary operator and a unilateral shift by Wold decomposition. By Lemma 2 of [24],

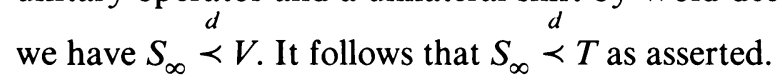

Lemma 2.3. Let $T$ be a contraction of analytic type. Then $\mu_{T}=\min \left\{\alpha: S_{\alpha} \stackrel{d}{\prec} T\right\}$.

Proof. In light of Lemma 2.2, we need only show that $\mu_{T} \leqslant n$ if and only if $S_{n} \stackrel{d}{\prec} T$, where $n=1,2, \ldots$. The proof that $\mu_{T} \leqslant n$ implies $S_{n} \stackrel{d}{\prec} T$ is essentially contained in the proof of (a) $\Rightarrow$ (b) in [1, Proposition 2.2]. That $S_{n} \stackrel{d}{\prec} T$ implies $\mu_{T} \leqslant n$ follows easily from Lemma 2.1(a) and the fact that $\mu_{S_{n}}=n$.

Recall that an operator $T$ is cyclic if $\mu_{T}=1$. The next corollary has been noted before by Takahashi [18, Lemma 3] for c.n.u. contractions.

COROLlaRY 2.4. Let $T$ be a contraction of analytic type and $S$ be the simple unilateral shift.

(a) $S \stackrel{d}{\prec} T$ if and only if $T$ is cyclic.

(b) $S \prec T$ if and only if $T$ is cyclic and not of class $C_{0}$.

TheOrem 2.5. Let $T$ on $H$ be a contraction of analytic type. Then there exist a $C_{._{0}}$ contraction $V$ with $d_{V^{*}}=\mu_{V}=\mu_{T}$ and a $C_{0}$. contraction $W$ with $d_{W}=\mu_{W^{*}}=\mu_{T^{*}}$ such that $V \prec T \prec W$.

Proof. Let $\alpha=\mu_{T}$ and $X: H_{\alpha}^{2} \rightarrow H$ be such that $X S_{\alpha}=T X$ and $\overline{X H_{\alpha}^{2}}=H$. Let $K=(\operatorname{ker} X)^{\perp}$ and $V=P_{K} S_{\alpha} \mid K$, where $P_{K}$ denotes the orthogonal projection onto $K$. Then $V$ is a $C_{._{0}}$ contraction and $V \prec T$. We have $\mu_{V} \leqslant d_{V^{*}}=\alpha=\mu_{T}$ (cf. [16]). On the other hand, $V \prec T$ implies that $\mu_{V} \geqslant \mu_{T}$. Therefore, $V$ satisfies $d_{V^{*}}=\mu_{V}=\mu_{T}$. Apply the above arguments to $T^{*}$ to obtain $W$.

The preceding theorem generalizes the main result in [16] from $C_{._{0}}$ contractions to contractions of analytic type. Finally, we turn to an arbitrary contraction.

TheOREM 2.6. Let $T$ be a contraction. Then $\mu_{T}=\min \left\{\mu_{V}: V\right.$ is an isometry and $V \stackrel{d}{\prec}\}$.

Proof. Let $T=U_{s} \oplus T^{\prime}$, where $U_{s}$ is a singular unitary operator and $T^{\prime}$ is of analytic type. From $\operatorname{Alg} T=\operatorname{Alg} U_{s} \oplus \operatorname{Alg} T^{\prime}$, we infer that $\mu_{T}=\max \left\{\mu_{U_{s}}, \mu_{T^{\prime}}\right\}$ (cf. [22, Lemma 1.3 and 23, Lemma 3]). Letting $\alpha=\mu_{T}$, we have $\mu_{U_{s}} \leqslant \alpha$ and $\mu_{T^{\prime}} \leqslant \alpha$. Lemma 2.3 implies that $S_{\alpha} \prec T^{\prime}$. Let $V=U_{s} \oplus S_{\alpha}$. Then $V$ is an isometry, $V \prec T$ and $\mu_{V}=\max \left\{\mu_{U_{s}}, \mu_{S_{\alpha}}\right\}=\alpha$. This shows that $\mu_{T} \geqslant \min \left\{\mu_{V}: V\right.$ is an isometry and $V \prec T\}$. 
For the reverse inequality, assume that $V$ is an isometry such that $V \stackrel{d}{\prec} T$. Let $V=U_{s}^{\prime} \oplus V^{\prime}$, where $U_{s}^{\prime}$ is a singular unitary operator and $V^{\prime}$ is of analytic type. Then $U_{s}^{\prime} \oplus V^{\prime} \stackrel{d}{\prec} U_{s} \oplus T^{\prime}$ implies that $U_{s}^{\prime} \stackrel{d}{\prec} U_{s}$ and $V^{\prime} \stackrel{d}{\prec} T^{\prime}$. Hence $\mu_{U_{s}^{\prime}} \geqslant \mu_{U_{s}}$ and $\mu_{V^{\prime}} \geqslant \mu_{T^{\prime}}$ by Lemma 2.1(a). Therefore, $\mu_{T}=\max \left\{\mu_{U_{s}}, \mu_{T^{\prime}}\right\} \leqslant \max \left\{\mu_{U_{s}^{\prime}}, \mu_{V^{\prime}}\right\}=\mu_{V}$. This completes the proof.

3. Hyponormal operators. In this section, we consider the problem when a hyponormal operator is quasisimilar to an isometry and, more generally, when two hyponormal contractions are quasisimilar. Our main results reduce these problems to the corresponding ones for their c.n.u. parts if these parts are of finite multiplicity.

We start by considering $C_{1}$. contractions. Let $T$ be such a contraction on $H$. It has been proved by Sz.-Nagy and Foiaş [17, Proposition II.3.5] that there exists an isometry $V$ such that $T \prec V$ and, furthermore, the intertwining quasiaffinity $X$ can be chosen such that $\|X h\|=\inf _{k \geqslant 0}\left\|T^{k} h\right\|$ for all $h$ in $H$. Following Alexander [1], we shall call this isometry $V$ the canonical isometry and $X$ the canonical intertwining quasiaffinity of $T$. Our first result says that the canonical isometry $V$ of a $C_{1}$. contraction $T$ is "maximal" with respect to the property $T \prec V$. It was essentially proved in [1, Theorem 2.8]. We include the proof here for easy reference.

Proposition 3.1. Let $T$ be a $C_{1}$. contraction with the canonical isometry $V$. If $T \stackrel{d}{\prec} V_{1}$

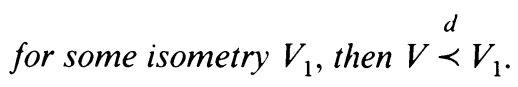

Proof. Assume that $T, V$ and $V_{1}$ are acting on the spaces $H, K$ and $L$, respectively. Let $Y: H \rightarrow L$ have dense range and satisfy $Y T=V_{1} Y$ and let $X: H \rightarrow K$ be the canonical intertwining quasiaffinity of $T$. Define $Z: K \rightarrow L$ by $Z X h=Y h$ for $h \in H$. Then $Z$ is densely defined and has dense range. We have

$$
\|Z X h\|=\|Y h\|=\left\|V_{1}^{k} Y h\right\|=\left\|Y T^{k} h\right\| \leqslant\|Y\|\left\|T^{k} h\right\|
$$

for any $k \geqslant 1$. It follows that

$$
\|Z X h\| \leqslant\|Y\| \inf _{k}\left\|T^{k} h\right\|=\|Y\|\|X h\|
$$

by the remark above. Thus $Z$ can be extended to a bounded operator on $K$. Since $Z V X h=Z X T h=Y T h=V_{1} Y h=V_{1} Z X h$ for any $h \in H$ and $\{X h: h \in H\}$ is dense in $K$, we infer that $Z V=V_{1} Z$. Thus $V \stackrel{d}{\prec} V_{1}$ as asserted.

Corollary 3.2. Let $T$ be a $C_{1}$. contraction with the canonical isometry $V$. Then $\mu_{V}=\sup \left\{\mu_{V_{1}}: V_{1}\right.$ is an isometry and $\left.T \prec V_{1}\right\}$.

In the following, we shall show that under certain circumstances the canonical isometry $V$ of a $C_{1}$. contraction $T$ is unique in the sense that $T \stackrel{d}{\sim} V_{1}$ for some isometry $V_{1}$ implies $V_{1} \cong V$. For this purpose, we need the following lemmas. 
LeMma 3.3. Let $A, B$ and $N$ be normal operators with $\mu_{N}<\infty$. If $A \oplus N \cong B \oplus N$, then $A \cong B$.

Proof. By the spectral theorem, $N$ is unitarily equivalent to a direct sum of finitely many cyclic normal operators, say, $N \cong N_{1} \oplus \cdots \oplus N_{k}$. Hence $A \oplus N_{1} \oplus$ $\cdots \oplus N_{k} \cong B \oplus N_{1} \oplus \cdots \oplus N_{k}$. A repeated use of [4, Proposition II.9.5] yields $A \cong B$.

LEMMA 3.4. For $j=1,2$, let $V_{j}=U_{j} \oplus S_{j}$ be an isometry, where $U_{j}$ is a unitary operator and $S_{j}$ is a unilateral shift.

(a) If $V_{1} \stackrel{d}{\sim} V_{2}$, then $S_{1} \cong S_{2}$.

(b) If $V_{1} \stackrel{d}{\sim} V_{2}$ and $\mu_{S_{1}}<\infty$, then $V_{1} \cong V_{2}$.

Proof. Assume that $V_{j}=U_{j} \oplus S_{j}$ is acting on $H_{j}=K_{j} \oplus L_{j}, j=1,2$. Let $X$ : $H_{1} \rightarrow H_{2}$ be an intertwining operator with dense range.

(a) Since $U_{1}$ is of class $C_{11}$ and $S_{2}$ is of class $C_{10}$, it is easily seen that $X K_{1} \subseteq K_{2}$. Let $Y=P_{L_{2}} X \mid L_{1}$, where $P_{L_{2}}$ denotes the orthogonal projection from $H_{2}$ onto $L_{2}$. Then $Y$ has dense range and $Y S_{1}=S_{2} Y$. This shows that $S_{1} \prec S_{2}$ whence $\mu_{S_{1}} \geqslant \mu_{S_{2}}$. By symmetry, we have $\mu_{S_{2}} \geqslant \mu_{S_{1}}$. Thus $\mu_{S_{1}}=\mu_{S_{2}}$. For unilateral shifts, this implies $S_{1} \cong S_{2}$.

(b) Let $W_{j}$ on $M_{j}$ be the minimal unitary extension of $S_{j}, j=1,2$. Then $U_{j} \oplus W_{j}$ is the minimal unitary extension of $V_{j}$. We can extend $X$ to an operator $Z: K_{1} \oplus M_{1} \rightarrow$ $K_{2} \oplus M_{2}$ which intertwines $U_{1} \oplus W_{1}$ and $U_{2} \oplus W_{2}$ and has dense range (cf. [7, Corollary 5.1]). Thus $U_{1} \oplus W_{1} \prec U_{2} \oplus W_{2}$. By [7, Lemma 4.1], $U_{2} \oplus W_{2}$ is unitarily equivalent to a direct summand of $U_{1} \oplus W_{1}$. Symmetrically, $U_{1} \oplus W_{1}$ is also unitarily equivalent to a direct summand of $U_{2} \oplus W_{2}$. We infer that $U_{1} \oplus W_{1} \cong U_{2}$ $\oplus W_{2}$ (cf. [13]). From (a), $S_{1} \cong S_{2}$ and therefore $W_{1} \cong W_{2}$. Since $\mu_{W_{1}}=\mu_{S_{1}}<\infty$, we conclude from Lemma 3.3 that $U_{1} \cong U_{2}$. This, together with $S_{1} \cong S_{2}$, yields $V_{1} \cong V_{2}$.

We remark that in Lemma 3.4(b), the assumption on $\mu_{S_{1}}$ is essential. As an example, let $V_{1}=U \oplus S_{\infty}$ and $V_{2}=S_{\infty}$, where $U$ is the bilateral shift. Then $V_{1} \stackrel{d}{\sim} V_{2}$ by Lemma 2.2 , but $V_{1}$ is not unitarily equivalent to $V_{2}$.

The next two propositions give the uniqueness property for the canonical isometry of a $C_{1}$. contraction under certain conditions. In particular, the first one partially generalizes Theorem 2.8 of [1].

Proposition 3.5. Let $T$ be a $C_{1}$. contraction with the canonical isometry $V$. Assume that $V=U \oplus S$, where $U$ is unitary and $S$ is a unilateral shift with $\mu_{S}<\infty$. If $T \stackrel{d}{\sim} V_{1}$ for some isometry $V_{1}$, then $V \cong V_{1}$.

Proof. We infer from Proposition 3.1 that $V \stackrel{d}{\prec} V_{1}$. On the other hand, we also have $V_{1} \stackrel{d}{\sim} T \prec V$. Thus $V \stackrel{d}{\sim} V_{1}$. Since $\mu_{S}<\infty$, the assertion follows from Lemma 3.4(b). 
Proposition 3.6. Let $T$ be a contraction. If $T \approx V_{1}$ for some isometry $V_{1}$, then $T$ is of class $C_{1}$. and $V_{1}$ is unitarily equivalent to the canonical isometry of $T$.

Proof. That $T$ is of class $C_{1}$. is trivial. Let $V$ be the canonical isometry and $X$ the canonical intertwining quasiaffinity of $T$. Let $Y$ be an invertible operator such that $Y T=V_{1} Y$. As proved in Proposition 3.1, there is an operator $Z$ with dense range such that $Z V=V_{1} Z$ and $Z X=Y$. This implies that $X$ is left invertible with left inverse $Y^{-1} Z$. Hence $X$ has a closed range and so is invertible. We have $V \approx T \approx V_{1}$. Since $V$ and $V_{1}$ are isometries, this implies $V \cong V_{1}$ by [12, Theorem 3.1].

We now come back to our original problem concerning the quasisimilarity of hyponormal contractions. The next result is the major tool to prove our main theorem in this section. It greatly generalizes Theorem 5 of [20], where only $C_{10}$ contractions with finite defect indices are considered.

THEOREM 3.7. Let $T$ be a $C_{1}$. contraction with $\mu_{T}<\infty$. If $X \in\{T\}^{\prime}$ has dense range, then $X$ is one-to-one.

Proof. Let $V$ be the canonical isometry and $Y$ the canonical intertwining quasiaffinity of $T$. Since $Y X$ has dense range and satisfies $Y X T=V Y X$, by Proposition 3.1 there exists an operator $Z$ with dense range such that $Z V=V Z$ and $Z Y=Y X$. Extend $Z$ to an operator $W$ commuting with the minimal unitary extension $U$ of $V$ (cf. [7, Corollary 5.1]). Since $Z$ has dense range, so does $W$. Note that $\mu_{W}=\mu_{V} \leqslant \mu_{T}<\infty$ (cf. [23, Lemma 4]). Hence $W$ is one-to-one and so is $Z$ (cf. [13]). From the injectivity of $Z Y=Y X$, we conclude that $X$ is one-to-one.

Note that in the preceding theorem, the assumption $\mu_{T}<\infty$ is essential. Example: $T=S \oplus S \oplus \cdots$ on $H=H^{2} \oplus H^{2} \oplus \cdots$, where $S$ denotes the simple unilateral shift on $H^{2}$, and $X$ in $\{T\}^{\prime}$ defined by $X\left(x_{1} \oplus x_{2} \oplus x_{3} \oplus \cdots\right)=x_{2} \oplus x_{3} \oplus \cdots$ on $H$.

Corollary 3.8. For $j=1,2$, let $T_{j}$ be a $C_{1}$. contraction with $\mu_{T_{j}}<\infty$. If $T_{1} \stackrel{d}{\sim} T_{2}$, then $T_{1} \sim T_{2}$.

Now we are ready for the main theorem of this section. It generalizes Theorem 6 of [20].

THEOREM 3.9. Let $T=T_{1} \oplus T_{2}$ and $S=S_{1} \oplus S_{2}$ be contractions, where $T_{1}$ and $S_{1}$ are of class $C_{11}, T_{2}$ and $S_{2}$ are of class $C_{._{0}}$ and $\mu_{T_{2}}<\infty$. Then $T \sim S$ if and only if $T_{1} \sim S_{1}$ and $T_{2} \sim S_{2}$.

Proof. Assume that $T \sim S$. Let

$$
T_{2}=\left[\begin{array}{cc}
T_{3} & * \\
0 & T_{4}
\end{array}\right] \text { and } S_{2}=\left[\begin{array}{cc}
S_{3} & * \\
0 & S_{4}
\end{array}\right]
$$

be the triangulations of type

$$
\left[\begin{array}{cc}
C_{00} & * \\
0 & C_{10}
\end{array}\right]
$$


(cf. [17, Theorem II.4.1]) and let $X$ be a quasiaffinity such that $X T=S X$. We have

$$
T=\left[\begin{array}{ccc}
T_{1} & 0 & 0 \\
0 & T_{3} & * \\
0 & 0 & T_{4}
\end{array}\right] \text { and } S=\left[\begin{array}{ccc}
S_{1} & 0 & 0 \\
0 & S_{3} & * \\
0 & 0 & S_{4}
\end{array}\right]
$$

Let $X=\left[X_{i j}\right]_{i, j=1}^{3}$ be the corresponding matrix representation. Since $T_{1}$ is of class $C_{11}$ and $S_{2}$ is of class $C_{.0}$, we can easily deduce that $X_{21}=0$ and $X_{31}=0$. Similarly, that $T_{3}$ is of class $C_{00}$ and $S_{1}$ and $S_{4}$ are of class $C_{1}$. yields $X_{12}=0$ and $X_{32}=0$. Therefore,

$$
X=\left[\begin{array}{ccc}
X_{11} & 0 & X_{13} \\
0 & X_{22} & X_{23} \\
0 & 0 & X_{33}
\end{array}\right]
$$

Since $X_{33}$ satisfies $X_{33} T_{4}=S_{4} X_{33}$ and has dense range, we have $T_{4} \stackrel{d}{\prec} S_{4}$. By symmetry, $S_{4} \stackrel{d}{\prec} T_{4}$ and so $T_{4} \stackrel{d}{\sim} S_{4}$. Since $\mu_{T_{4}} \leqslant \mu_{T_{2}}<\infty$, we may apply Corollary 3.8 to conclude that $X_{33}$ is one-to-one. On the other hand, $X$ is a quasiaffinity implies that $X_{22}$ is one-to-one and

$$
\left[\begin{array}{cc}
X_{22} & X_{23} \\
0 & X_{33}
\end{array}\right]
$$

has dense range. It follows that

$$
\left[\begin{array}{cc}
X_{22} & X_{23} \\
0 & X_{33}
\end{array}\right]
$$

is a quasiaffinity and thus $T_{2} \prec S_{2}$. By symmetry, $S_{2} \prec T_{2}$ whence $T_{2} \sim S_{2}$.

As for $T_{1}$ and $S_{1}$, we note that $X_{11}$ is one-to-one and satisfies $X_{11} T_{1}=S_{1} X_{11}$. Since the $C_{11}$ contractions $T_{1}$ and $S_{1}$ are quasisimilar to unitary operators, say, $T_{1}^{\prime}$ and $S_{1}^{\prime}$ (cf. [17, Proposition II.3.5]), by [7, Lemma 4.1] $T_{1}^{\prime} \stackrel{d}{\sim} S_{1}^{\prime}$ implies that $T_{1}^{\prime}$ is unitarily equivalent to a direct summand of $S_{1}^{\prime}$. By symmetry, $S_{1}^{\prime}$ is unitarily equivalent to a direct summand of $T_{1}^{\prime}$. Therefore $T_{1}^{\prime} \cong S_{1}^{\prime}$ (cf. [13]) and thus $T_{1} \sim S_{1}$.

Corollary 3.10. Let $T$ and $S$ be hyponormal contractions. Assume that the c.n.u. part of $T$ has finite multiplicity. Then $T \sim S$ if and only if their unitary parts are unitarily equivalent and c.n.u. parts are quasisimilar to each other.

Proof. The assertion follows immediately from Theorem 3.9 and the fact that c.n.u. hyponormal contractions are of class $C_{.0}$ (cf. [15]).

Recall that any operator can be decomposed as the direct sum of a normal operator (its normal part) and a completely nonnormal operator (its c.n.n. part) (cf. [14, Corollary 1.3]).

COROllary 3.11. Let $T$ be a hyponormal operator whose c.n.n. part has finite multiplicity. Then $T$ is quasisimilar to an isometry if and only if its normal part is unitary and its c.n.n. part is quasisimilar to a unilateral shift. 
Proof. Assume that $T=T_{1} \oplus T_{2}$ is quasisimilar to the isometry $V=U \oplus S$, where $T_{1}$ is normal, $T_{2}$ is c.n.n., $U$ is unitary and $S$ is a unilateral shift. By Proposition 3.5 of [11], $T_{1}$ is unitarily equivalent to $U$ whence unitary. On the other hand, by Clary's result [2], $T$ and $V$ have equal spectra. Thus $\|T\|=r(T)=r(V)=1$, where $r(T)$ and $r(V)$ are the spectral radii of $T$ and $V$ (cf. [9, Problem 205]). $T_{2} \sim S$ follows from Corollary 3.10 .

Corollary 3.10 generalizes a previous result of the author's for hyponormal contractions with finite defect indices [20, Corollary 7] and Hastings' for subnormal contractions and isometries [10]; Corollary 3.11 generalizes Hastings' for subnormal operators [11, Corollary to Theorem 4.5]. Also note that the preceding three results may not hold if there is no finiteness assumption on the multiplicity; an example was given in [11].

We close this section by mentioning the following conjecture. It has been verified for cyclic subnormal operators by Conway [3, Proposition 2.5] and quasinormal operators by Williams [19, Theorem 4].

Conjecture 3.12. Let $T$ and $S$ be hyponormal operators. Assume that the c.n.n. part of $T$ has finite multiplicity. Then $T \sim S$ if and only if their normal parts are unitarily equivalent and their c.n.n. parts are quasisimilar to each other.

4. Quasinormal and subnormal operators. In this section, we consider the more restrictive classes of quasinormal and subnormal operators. In certain circumstances, such an operator quasisimilar or similar to an isometry may even be unitarily equivalent to it, thus strengthening the results in $\$ 3$. More precisely, we show that a quasinormal operator quasisimilar to an isometry is unitarily equivalent to it (Proposition 4.2) and a subnormal operator $T$ similar to an isometry $V$ and belonging to $\mathrm{Alg} V$ is unitarily equivalent to $V$ (Proposition 4.6). It is interesting to contrast these positive results with the known negative ones: (1) there are quasisimilar c.n.n. quasinormal operators which are not similar to each other [19, Example 2]; (2) there are c.n.n. subnormal operators similar to a simple unilateral shift without being unitarily equivalent to it [9, Solution 199]. We first consider quasinormal operators and start with the following lemma whose proof is left to the readers.

LemMA 4.1. Let $T$ be a quasinormal operator. Then $T=T_{1} \oplus T_{2} \oplus T_{3}$, where $T_{1}$ is normal, $T_{2}$ is c.n.u. and satisfies $\operatorname{ker}\left(1-T_{2}{ }^{*} T_{2}\right)=\{0\}$ and $T_{3}$ is a unilateral shift.

In particular, if $T$ is a quasinormal contraction decomposed as above, then $T_{2}$ is of class $C_{00}[6$, Corollaries $2.5,2.6]$ whence $\left[{ }_{0}^{T_{2}}{ }_{T_{3}}\right]$ is the triangulation of type $\left[{ }_{0}^{C_{(0)}}{ }_{C_{10}}^{*}\right]$. Using this decomposition, we can prove the following result which generalizes the fact that quasisimilar isometries are unitarily equivalent [12, Theorem 3.1].

Proposition 4.2. Let $T$ be a quasinormal operator and $V$ be an isometry. If $T \sim V$, then $T \cong V$.

Proof. Let $T=T_{1} \oplus T_{2} \oplus T_{3}$ be as in Lemma 4.1 and $V=U \oplus S$, where $U$ is unitary and $S$ is a unilateral shift. Let $X$ be a quasiaffinity such that $X T=V X$. By [11, Proposition 3.5], $T_{1} \cong U$ is unitary and, as proved in Corollary 3.11, $T$ is a 
contraction. We infer, as in the proof of Theorem 3.9, that $X$ has the matrix representation

$$
X=\left[\begin{array}{ccc}
X_{11} & 0 & X_{13} \\
0 & 0 & X_{23}
\end{array}\right]
$$

It follows from the injectivity of $X$ that $T_{2}$ is acting on the zero space $\{0\}$. Hence $T=T_{1} \oplus T_{3}$ is an isometry quasisimilar to $V$. We conclude that $T \cong V$, completing the proof.

The forerunner of our last proposition is a result of Conway's [5]: If $T$ is an operator in $\operatorname{Alg} S$, the weakly closed algebra generated by the simple unilateral shift $S$ and $I$, and $T \sim S$, then $T \cong S$. We would like to generalize this with $S$ replaced by an arbitrary isometry $V$ of which we are successful only under the stronger condition that $T$ be similar to $V$. We start with the following lemma which disposes of the trivial case when $V$ is unitary.

LEMMA 4.3. Let $N$ be a normal operator and $T \in \operatorname{Alg} N$. If $T \stackrel{d}{\sim} N$, then $T \cong N$.

Proof. Since $T$ is normal, the conclusion follows from [7, Lemma 4.1 and 13].

For $\varphi$ in $L^{\infty}$ of the unit circle, let $T_{\varphi}$ denote the Toeplitz operator defined by $T_{\varphi} f=P(\varphi f)$ for $f \in H^{2}$, where $P$ denotes the orthogonal projection from $L^{2}$ onto $H^{2}$. Since Alg $S=\left\{\varphi(S): \varphi \in H^{\infty}\right\}$ consists of analytic Toeplitz operators [9, Problem 148], where $S$ is the simple unilateral shift, we next show that such an operator is c.n.n. if $\varphi$ is not a constant function. Goor [8] has shown before that any Toeplitz contraction $T_{\varphi}$ with a nonconstant $\varphi$ is c.n.u. Our proof is based on his. It also follows from [4, Corollary VIII. 2.14].

Lemma 4.4. Let $T_{\varphi}$ be an analytic Toeplitz operator, where $\varphi \in H^{\infty}$. Then the following statements are equivalent:

(a) $\varphi$ is not constant;

(b) $T_{\varphi}$ is not normal;

(c) $T_{\varphi}$ is c.n.n.

Proof. The equivalence of (a) and (b) is well known. We only prove (a) $\Rightarrow(c)$. Assume that $T_{\varphi}$ is a contraction. Let $K \subseteq H^{2}$ be such that $T_{\varphi} \mid K$ is the normal part of $T_{\varphi}$. By [14, Corollary 1.3], $K=\left\{f \in H^{2}: T_{\varphi}^{m} T_{\bar{\varphi}}^{n} f=T_{\bar{\varphi}}^{n} T_{\varphi}^{m} f \forall m, n \geqslant 1\right\}$. On the other hand, if $L \subseteq H^{2}$ is such that $T_{\varphi} \mid L$ is the unitary part of $T_{\varphi}$, then, as proved by Goor [8], $L=\left\{f \in H^{2}: \varphi^{n} f, \bar{\varphi}^{n} f \in H^{2} \forall n \geqslant 1\right\}$. Obviously, we have $L \subseteq K$. For the converse, let $f \in K$. We have

$$
\left\|\bar{\varphi}^{n} f\right\|=\left\|\phi^{n} f\right\|=\left\|T_{\varphi}^{n} f\right\|=\left\|T_{\bar{\varphi}}^{n} f\right\|=\left\|P\left(\bar{\varphi}^{n} f\right)\right\| \leqslant\left\|\bar{\varphi}^{n} f\right\|
$$

for any $n \geqslant 1$. Hence $\left\|P\left(\bar{\varphi}^{n} f\right)\right\|=\left\|\bar{\varphi}^{n} f\right\|$ and it follows that $\bar{\varphi}^{n} f \in H^{2}$ for all $n$. This shows that $K \subseteq L$, and thus $L=K$. Since $\varphi$ is not a constant function, we have $L=\{0\}$ by $[8]$. Therefore $K=\{0\}$ and $T_{\varphi}$ is c.n.n.

LEMMA 4.5. A direct sum of operators is c.n.n. if and only if all its summands are c.n.n. 
Proof. It follows from [14, Corollary 1.3] that the normal part of the direct sum is the direct sum of the normal parts of the summands. Our assertion follows immediately.

Now we are ready for our last result.

Proposition 4.6. Let $V$ be an isometry. If $T \in A \operatorname{Ag} V$ and $T \approx V$, then $T \cong V$.

Proof. Let $V=U_{s} \oplus U_{a} \oplus S$, where $U_{s}$ and $U_{a}$ are singular and absolutely continuous unitary operators and $S$ is a unilateral shift. In view of Lemma 4.3, we may assume that $V$ is not unitary. Hence $T \in \operatorname{Alg} V$ implies that $T=W \oplus$ $\varphi\left(U_{a} \oplus S\right)$, where $W \in \operatorname{Alg} U_{s}$ and $\varphi \in H^{\infty}$ (cf. [22, Lemma 1.3 and 21, Lemma 3.11]). Note that $\varphi$ is not constant. Indeed, if it is, then $T$ is normal whence $T \approx V$ implies that $T \cong U_{s} \oplus U_{a}$ by [11, Proposition 3.5]. It follows from $U_{s} \oplus U_{a} \approx V$ that $V$ is unitary, contradicting our assumption. Hence $\varphi(S)$ is c.n.n. by Lemmas 4.4 and 4.5. Therefore $T \approx V$ implies that $W \oplus \varphi\left(U_{a}\right) \cong U_{s} \oplus U_{a}$ and $\varphi(S) \approx S$ (cf. [3, Proposition 2.6]). To complete the proof, we need only show that $\varphi(S) \cong S$.

For any operator $A$, let $\sigma(A)$ and $\sigma_{\text {ap }}(A)$ denote its spectrum and approximate point spectrum, respectively. Moreover, let $S_{0}$ denote the simple unilateral shift and $M_{\varphi}$ the operator of multiplication by $\varphi$ on $L^{2}$ of the unit circle. We have $\sigma\left(M_{\varphi}\right)=$ $\sigma_{\text {ap }}\left(M_{\varphi}\right)=\sigma_{\text {ap }}\left(\varphi\left(S_{0}\right)\right)=\sigma_{\text {ap }}(\varphi(S))=\sigma_{\text {ap }}(S)=\{z:|z|=1\}$ (cf. [9, Problem 245]). Hence $M_{\varphi}$ is unitary and therefore $\varphi$ is an inner function. Thus $\varphi(S)$ is an isometry which is similar to $S$. We conclude that $\varphi(S) \cong S$, completing the proof.

To conclude this paper, we propose the following conjecture.

Conjecture 4.7. Let $V$ be an isometry. If $T \in \operatorname{Alg} V$ and $T \sim V$, then $T \cong V$.

\section{REFERENCES}

1. V. T. Alexander, Contraction operators quasisimilar to a unilateral shift, Trans. Amer. Math. Soc. 283 (1984), 697-703.

2. S. Clary, Equality of spectra of quasi-similar hyponormal operators, Proc. Amer. Math. Soc. 53 (1975), $88-90$.

3. J. B. Conway, On quasisimilarity for subnormal operators, Illinois J. Math. 24 (1980), 689-702.

4. __ Subnormal operators, Pitman, London, 1981.

5. On quasisimilarity for subnormal operators. II, Canad. Math. Bull. 25 (1982), 37-40.

6. J. B. Conway and P. Y. Wu, The structure of quasinormal operators and the double commutant property, Trans. Amer. Math Soc. 270 (1982), 641-657.

7. R. G. Douglas, On the operator equation $S^{*} X T=X$ and related topics, Acta Sci. Math. (Szeged) 30 (1969), 19-32.

8. R. Goor, On Toeplitz operators which are contractions, Proc. Amer. Math. Soc. 34 (1972), $191-192$.

9. P. R. Halmos, A Hilbert space problem book, 2nd ed., Springer-Verlag, New York, 1982.

10. W. W. Hastings, Subnormal operators quasisimilar to an isometry, Notices Amer. Math. Soc. 22 (1975), A-189.

11. . Subnormal operators quasisimilar to an isometry, Trans. Amer. Math. Soc. 256 (1979), $145-161$.

12. T. B. Hoover, Quasi-similarity of operators, Illinois J. Math. 16 (1972), 678-686.

13. R. V. Kadison and I. M. Singer, Three test problems in operator theory, Pacific J. Math. 7 (1957), $1101-1106$.

14. B. B. Morrel, $A$ decomposition for some operators, Indiana Univ. Math. J. 23 (1973), 497-511.

15. C. R. Putnam, Hyponormal contractions and strong power convergence, Pacific J. Math. 57 (1975), $531-538$

16. B. Sz.-Nagy and C. Foiaş, Vecteurs cycliques et quasiaffinités, Studia Math. 31 (1968), 35-42.

17. Harmonic analysis of operators on Hilbert space, North-Holland, Amsterdam, 1970. 
18. K. Takahashi, On the reflexivity of some contractions (preprint).

19. L. R. Williams, Equality of essential spectra of quasisimilar quasinormal operators, J. Operator Theory 3 (1980), 57-69.

20. P. Y. Wu, On the quasi-similarity of hyponormal contractions, Illinois J. Math. 25 (1981), 498-503.

21. $137-149$.

22. 209-217. , Approximate decompositions of certain contractions, Acta Sci. Math. (Szeged) 44 (1982), , On the reflexivity of $C_{1}$. contractions and weak contractions, J. Operator Theory 8 (1982),

24. , Multiplicities of isometries, Integral Equations Operator Theory 7 (1984), 436-439.

Theory 7 (1984), 899-904.

Department of Applied Mathematics, National Chiao Tung University, Hsinchu, Taiwan, REPUBlic OF China 